\title{
Modeling Green Fabs - A Queuing Theory Approach for Evaluating Energy Performance
}

\author{
Hyun Woo Jeon and Vittaldas V. Prabhu \\ Harold and Inge Marcus Department of Industrial and Manufacturing Engineering, \\ Pennsylvania State University, University Park, PA 16802 USA \\ albert.jeon@psu.edu, prabhu@engr.psu.edu
}

\begin{abstract}
More than $30 \%$ of the total energy consumed in the U.S. is attributed to industrial sector which motivated improvements in energy efficiency of manufacturing processes and entire factories. Semiconductor fabrication (fab) represents an interesting challenge for energy efficiency because of their relatively high energy consumption to process a unit mass of material. The focus of this paper is to develop an energy-aware analytical model based on queuing theory that has re-entrant network structure commonly found in fabs to analyze the impact of reducing idle power consumption in individual equipment. The proposed analytical model based on BCMP network for re-entrant lines has the same mathematical form as serial lines and is tested for using detailed simulation of a generic CMOS fab with three processing steps. Results show that the energy consumption predicted by the analytical model differs from simulation typically within $10 \%$ and worst case of $14 \%$, in the tested cases.
\end{abstract}

Keywords: Sustainability, Queueing Network, Re-entrant Networks, Semiconductor Manufacturing, Energy-aware Model.

\section{Introduction}

Faced with a growing trend of increasing energy consumption worldwide, various organizations are trying to be more energy efficient [1]. Especially there is a particular need for the U.S., as one of the largest energy consumers among nations, to increase energy efficiency across all segments of the economy as the American industrial segment accounts for $31 \%$ of its total energy consumption [2]. Two benefits that can be expected from improved energy efficiency in the industry segment are direct cost savings and indirect environmental benefit, both stemming from reduced energy consumption. Consequently there has been increasing research on understanding energy consumption of various manufacturing processes and associated equipment [3]. This prior research has found that semiconductor manufacturing processes is among the highest energy consumption per unit mass in the manufacturing industry. For example, the oxidation process in fabs typically consumes $1 . \mathrm{E}+13-14 \mathrm{~J} / \mathrm{kg}$ compared to milling steel which consumes about $1 . \mathrm{E}+05 \mathrm{~J} / \mathrm{kg}$. Moreover, idling manufacturing machines typically consume as much as 40 to $60 \%$ of the power when they are busy for processing parts [4]. A logical progression of research effort is to build on the energy consumption models of unit processes to higher 
levels such as the factory level and the supply chain level. For analyzing busy/idle states of a system consisting of many machines, simulation and queuing modeling are attractive approaches. Good insights can be acquired when both approaches are used, including testing and validation of each other judiciously [5].

Our recent efforts in this direction include developing hybrid simulation models consisting of continuous and discrete process for predicting energy consumption in discrete manufacturing [6]. We have also proposed queuing models to predict energy savings in serial production lines when idling machines are switched to a lower power state in serial production lines consisting of machines with Poisson arrival and exponential service time [7].

This paper builds on our previous work [7] to extend and generalize the energyaware queuing model to a re-entrant structure which is applied to model semiconductor fabs. Section 2 reviews the prior analytical model along with its parameters and limitations. In Section 3 a model for re-entrant lines is introduced and energy performance characteristics are derived. In Section 4 a generic semiconductor production system is used for comparing results predicted by the analytical model with those by detailed simulation. Section 5 summarizes this paper with possible future research directions.

\section{Energy-Aware Queuing Model}

Our recently proposed approach for improving energy efficiency in manufacturing system is to use energy control policies that switch machines to a lower power consumption state when a machine is anticipated to be idle for longer than some threshold, $\tau[6,7]$. In order to predict the efficiency gained by using such energy control policies, the state transitions between the various energy consumption states and production states of the machines need to be modeled. Without any energy control policy $\left(E C_{0}\right)$ the machine power consumption in its idle state will be $W_{1}$, the nominal power for idling. When energy control policy is used $\left(E C_{1}\right)$, energy state will be switched to the low power idling of $W_{0}$ if the idle time duration is anticipated to be longer than $\tau$; otherwise it will be $W_{1}$. In the busy state the power consumption will be $W_{p}$ regardless of whether $E C_{0}$ or $E C_{1}$ used.

For an M/M/1 queue with an arrival rate of $\lambda$ and a service rate $\mu$, the probability that a new arrival arrives after the time threshold $\tau$ is given by

$$
P(x>\tau)=\int_{\tau}^{\infty} \lambda e^{-\lambda x} d x=e^{-\lambda \tau}
$$

Based on this, the total energy consumption with $E C_{0}$ and $E C_{1}$ over time $T$ can be modeled as follows

$$
\begin{gathered}
E_{E C 0}=\left\{W_{p} \rho+W_{1}(1-\rho)\right\} T \\
E_{E C 1}=\left\{W_{p} \rho+W_{1}(1-\rho)\left(1-e^{-\lambda \tau}\right)+W_{0}(1-\rho) e^{-\lambda \tau}\right\} T
\end{gathered}
$$

In Equations 2 and $3 \rho$ is the machine utilization. Therefore the total energy saving can be expressed as

$$
E_{\text {total energy saved }}=\left(W_{1}-W_{0}\right)(1-\rho) e^{-\lambda \tau} T
$$


It should be noted from Equation 4 that energy saving can be increased by decreasing $W_{0}$ and $\tau$ for a given $\rho$. The above model for a single queue can be extended to serial production lines [7].

\section{$3 \quad$ Re-entrant System Modeling}

\subsection{Queueing Model for Re-entrant System}

Semiconductor fabs are markedly different from serial production lines or job shops $[8,9]$. A typical semiconductor fab has a re-entrant structure in which the products flow through a fixed route visiting a machine multiple times $[10,11]$. Semiconductor fabs can be modeled as an open queuing network in which parts visit a machine multiple times. It should be pointed out that because the route in semiconductor fab is deterministic such systems cannot be modeled as Jackson networks [12]. Therefore in this paper we will model semiconductor fabs as a BCMP network, which is also the approach taken by several other researchers [9, 11, 13]. The main parameters and assumptions in modeling a semiconductor fab as a BCMP queuing network are as follows

- Arrival rate from outside is $\lambda$ and $V_{m}$ is the number of visits to machine $m$ before a part exits the system.

- Arrival process is Poisson and mean service time follows exponential distribution with rates of $\lambda_{m}$ and $\mu_{m}$, respectively.

- Queueing discipline is FCFS (first come first serve) and parts are served regardless of buffer in which the part is located.

- Service rate $\mu_{m}$ is same for all parts in any buffer of the machine.

- Route of each part is fixed and deterministic.

- Idle time threshold for $E C_{1}$ is $\tau_{m}$ for machine $m$.

Based on the above model, the arrival rate at machine $m$ can be expressed as

$$
\lambda_{m}=V_{m} \lambda
$$

The utilization of machine $m$ can be also defined as

$$
\rho_{m}=\lambda_{m} / \mu_{m}=V_{m} \lambda / \mu_{m}
$$

The long run probability that there are $\mathrm{n}$ parts at a machine $m$ is therefore given by

$$
\pi_{n}(m)=\left(1-\rho_{m}\right) \rho_{m}^{n}
$$

From (6), (7) the probability of machine $m$ is busy, $P_{B}(m)$ can be expressed as

$$
P_{B}(m)=\rho_{m}
$$

The re-entrant structure of the model gives rise to multiple arrival streams at a machine that may not be independent of each other. Furthermore, summing these arrivals may not be an exact model for a composite Poisson process with an exponentially distributed inter-arrival time [14]. However treating the composite arrivals as a Poisson process can be a reasonable approximation [15], which we adopt for modeling the 
probability of a machine being in nominal power idling $\left(P_{N}\right)$ and low-power idling $\left(P_{L}\right)$ states when $E C_{1}$ is used. These probabilities for machine $m$ are

$$
\begin{gathered}
P_{N}(m) \approx\left(1-\rho_{m}\right)\left(1-e^{-\lambda_{m} \tau_{m}}\right) \\
P_{L}(m) \approx\left(1-\rho_{m}\right) e^{-\lambda_{m} \tau_{m}}
\end{gathered}
$$

From (8)-(10), the energy consumption for machine $m$ of a re-entrant system for time period $T$ can be modeled as

$$
\begin{gathered}
E_{E C 0}(m) \approx\left\{W_{p}(m) \rho_{m}+W_{1}(m)\left(1-\rho_{m}\right)\right\} T \\
E_{E C 1}(m) \approx\left\{\begin{array}{c}
W_{p}(m) \rho_{m}+W_{1}(m)\left(1-\rho_{m}\right)\left(1-e^{-\lambda_{m} \tau_{m}}\right) \\
+W_{0}(m)\left(1-\rho_{m}\right) e^{-\lambda_{m} \tau_{m}}
\end{array}\right\} T
\end{gathered}
$$

where $W_{p}(m), W_{1}(m)$, and $W_{0}(m)$ are power consumption levels of machine $m$ for busy, nominal power idling, and low power idling states respectively. It should be emphasized that this energy model for a re-entrant line has the same mathematical structure as the $\mathrm{M} / \mathrm{M} / 1$ model reviewed in the previous section. Therefore the total spent energy in the entire re-entrant system can be modeled as

$$
\begin{aligned}
& E_{\text {Total }(E C 0)} \approx \sum_{m=1}^{n} E_{E C 0}(m) \\
& E_{\text {Total }(E C 1)} \approx \sum_{m=1}^{n} E_{E C 1}(m)
\end{aligned}
$$

In the next section the energy consumption predicted by this analytical model is compared with a detailed simulation model to the efficacy of the model.

\subsection{Simulation Experiments}

Figure 1 illustrates a generic CMOS (Complementary Metal Oxide Semiconductor) fab with Deposition (D), Lithography (L), and Etching (E) processes and a re-entrant structure that follows an overall processing sequence indicated by the numerical values on the arrowed lines. The power consumption levels in these processes are hypothesized based on published data [16] and shown in Table 1 along with other parameters used to simulate three different scenarios with varying arrival rates into the system.

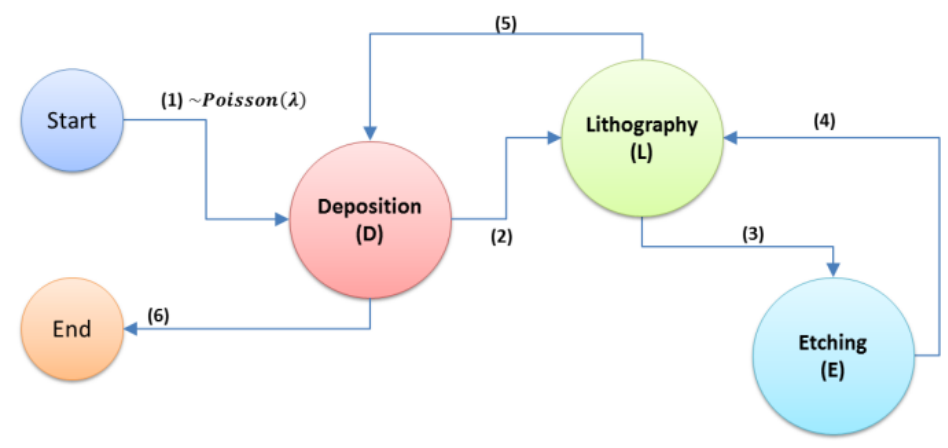

Fig. 1. Example re-entrant system for CMOS 
The simulation model was implemented by using the SIMIO simulation software package. For each scenario, $P_{B}, P_{N}$, and $P_{L}$ are estimated by fraction of the time a machine is in the corresponding state during a simulation run of 10,000 time units and averaged over 30 replications which is then used to compute the results in Tables 2 and 3 .

Table 1. Simulation parameters

\begin{tabular}{|c|c|c|c|c|c|c|c|c|c|}
\hline \multicolumn{10}{|c|}{ Parameters for each Simulation Scenario } \\
\hline Scenario & & 1 & & & 2 & & & 3 & \\
\hline Process & D & $\mathrm{L}$ & E & D & $\mathrm{L}$ & E & D & $\mathrm{L}$ & E \\
\hline$\lambda$ & 0.050 & 0.050 & 0.025 & 0.040 & 0.040 & 0.020 & 0.030 & 0.030 & 0.015 \\
\hline$\mu$ & 0.100 & 0.067 & 0.029 & 0.100 & 0.067 & 0.029 & 0.100 & 0.067 & 0.029 \\
\hline$\rho$ & 0.500 & 0.750 & 0.875 & 0.400 & 0.600 & 0.700 & 0.300 & 0.450 & 0.525 \\
\hline$\tau$ & 8.00 & 10.00 & 24.00 & 10.00 & 12.50 & 30.00 & 13.33 & 16.67 & 40.00 \\
\hline$\lambda \tau$ & 0.40 & 0.50 & 0.60 & 0.40 & 0.50 & 0.60 & 0.40 & 0.50 & 0.60 \\
\hline$W_{p}$ & 24857 & 9140 & 12238 & 24857 & 9140 & 12238 & 24857 & 9140 & 12238 \\
\hline$W_{1}$ & 8286 & 3047 & 4079 & 8286 & 3047 & 4079 & 8286 & 3047 & 4079 \\
\hline$W_{0}$ & 1657 & 609.3 & 815.9 & 1657 & 609.3 & 815.9 & 1657 & 609.3 & 815.9 \\
\hline Replication & & 30 & & & 30 & & & 30 & \\
\hline
\end{tabular}

The Figure 2 illustrates energy consumed with $E C_{0}$ and $E C_{1}$ of each of the deposition process in scenario 1 . As expected, energy savings in all three processes increases with time. Figure 3 shows the power consumption for the deposition processing step with $E C_{0}$ and $E C_{1}$ in scenario 1.

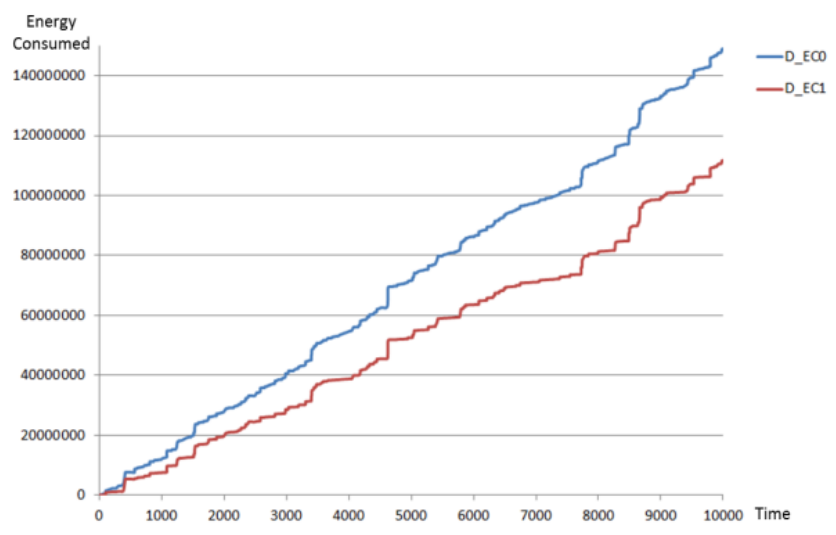

Fig. 2. Energy consumed in the deposition process in scenario 1

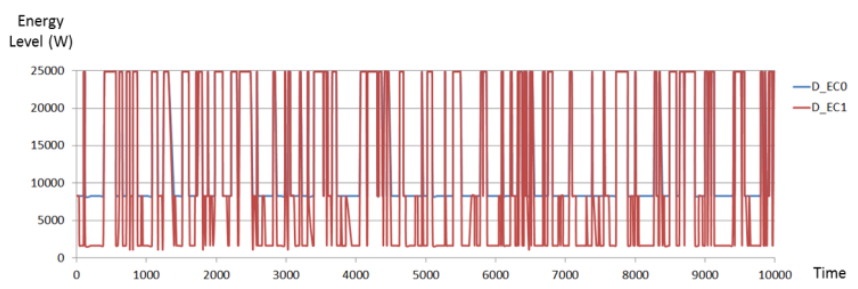

Fig. 3. Power consumption level for deposition process in scenario 1 


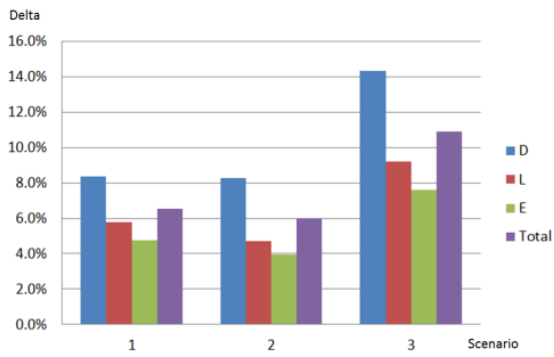

Fig. 4. Model difference with $E C_{1}$

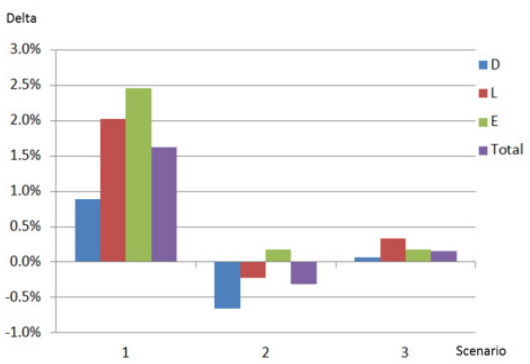

Fig. 5. Model difference with $E C_{0}$

Table 2. Comparison of Simulation and Analytical Models with $E C_{1}$

\begin{tabular}{ccccccccccccc}
\hline Scenario & \multicolumn{3}{c}{ Simulation } & \multicolumn{4}{c}{ Analytical Approximation } & \multicolumn{3}{c}{ Delta = (A-S)/S } \\
\hline & D & L & E & Total & D & L & E & Total & D & L & E & Total \\
1 & 13245 & 6850 & 10494 & 30590 & 14350 & 7247 & 10994 & 32591 & $8.3 \%$ & $5.8 \%$ & $4.8 \%$ & $6.5 \%$ \\
2 & 11315 & 5837 & 8903 & 26054 & 12248 & 6111 & 9253 & 27613 & $8.2 \%$ & $4.7 \%$ & $3.9 \%$ & $6.0 \%$ \\
3 & 8876 & 4556 & 6982 & 20414 & 10147 & 4976 & 7512 & 22634 & $14.3 \%$ & $9.2 \%$ & $7.6 \%$ & $10.9 \%$ \\
\hline
\end{tabular}

Table 3. Comparison of Simulation and Analytical Models with $E C_{0}$

\begin{tabular}{ccccccccccccc}
\hline Scenario & \multicolumn{4}{c}{ Simulation } & \multicolumn{4}{c}{ Analytical Approximation } & \multicolumn{3}{c}{ Delta $=(\mathbf{A - S}) / \mathbf{S}$} \\
\hline & $\mathrm{D}$ & $\mathrm{L}$ & $\mathrm{E}$ & Total & $\mathrm{D}$ & $\mathrm{L}$ & $\mathrm{E}$ & Total & $\mathrm{D}$ & $\mathrm{L}$ & $\mathrm{E}$ & Total \\
1 & 16425 & 7465 & 10949 & 34839 & 16571 & 7617 & 11218 & 35406 & $0.9 \%$ & $2.0 \%$ & $2.5 \%$ & $1.6 \%$ \\
2 & 15013 & 6717 & 9774 & 31504 & 14914 & 6703 & 9790 & 31407 & $-0.7 \%$ & $-0.2 \%$ & $0.2 \%$ & $-0.3 \%$ \\
3 & 13248 & 5769 & 8348 & 27365 & 13257 & 5789 & 8363 & 27408 & $0.1 \%$ & $0.3 \%$ & $0.2 \%$ & $0.2 \%$ \\
\hline
\end{tabular}

From Table 2 and 3, it can be observed that analytical and simulation estimate of total energy consumed typically differ within $10 \%$ with the worst case being $14.3 \%$. Figures 4 and 5 graphically illustrate the difference between analytical and simulation models (delta) presented in Tables 2 and 3. The difference is much less with $E C_{0}$ compared to $E C_{1}$ and delta decreases as utilization $\rho$ increases. We conjecture that the exponential distribution assumption for the composite inter-arrival times used in the analytical model underestimates the probability of being in low-power idling states, therefore overestimating the energy consumption. From energy efficiency perspective, $E C_{1}$ consumes less energy than $E C_{0}$ as expected. Energy savings $\left(E C_{0}-E C_{1}\right)$ decrease as $\rho$ increases because there is lesser opportunity for $E C_{1}$ as 
machines get busier. Moreover, for a given $\rho$, smaller $\tau$ will make $E C_{1}$ more energy efficient. In practice, the choice of $\tau$ will be constrained by the physics and economics of the process.

\section{Conclusions}

An energy-aware analytical model based on BCMP network is proposed in this paper for semiconductor fabs with re-entrant structure commonly. Focus of the analysis is to assess the energy efficiency gained by reducing idle power consumption by switching machines to a lower power state when idling. Simulation tests indicate that the energy consumption predicted by the analytical models are typically differ within $10 \%$ from detailed simulations with worst case being about 14\%, which makes the approach promising for rapid evaluation of candidate energy control strategies. Future work could focus on analyzing the factors that can influence the accuracy of the analytical model including the composite inter-arrival distribution model. Another potential future direction is to generalize the queuing networks to include G/G/1 and GI/G/1 systems $[17,18]$.

\section{References}

1. The U.S. Energy Information Administration: International Energy Outlook 2011, DOE/EIA-0484(2011) (2011), http: / /www.eia.gov/forecasts / ieo/

2. The U.S. Department of Energy: Annual Energy Review 2010, DOE/EIA-0384 (2010), http://www.eia.gov/totalenergy/data/annual/index.cfm

3. Gutowski, T.G., Branham, M.S., Dahmus, J.B., Jones, A.J., Thiriez, A., Sekulic, D.P.: Thermodynamic analysis of resources used in manufacturing processes. Environ. Sci. and Technol. 43, 1584-1590 (2009)

4. Diaz, N., Redelsheimer, E., Dornfeld, D.: Energy Consumption Characterization and Reduction Strategies for Milling Machine Tool Use. In: Glocalized Solutions for Sustainability in Manufacturing 2011, pp. 263-267 (2011)

5. Connors, D.P., Feigin, G.E., Tao, D.D.: A Queueing Network Model for Semiconductor Manufacturing. IEEE Transactions on Semiconductor Manufacturing 9(3) (1996)

6. Prabhu, V., Taisch, M.: Simulation Modeling of Energy Dynamics in Discrete Manufacturing Systems. In: Proceedings of 14th IFAC Symposium on Information Control Problems in Manufacturing (INCOM 2012), Bucharest, Romania, May 23-25 (2012)

7. Prabhu, V., Jeon, H.W., Taisch, M.: Modeling Green Factory Physics - An Analytical Approach. In: Proceedings of IEEE CASE 2012 (2012)

8. Graves, S.C.: A review of production scheduling. Operations Research 29, 646-675 (1981)

9. Kumar, P.R.: Re-entrant lines. Queueing Systems 13, 87-110 (1993)

10. Jaeger, R.C.: Introduction to Microelectronic Fabrication, 2nd edn., vol. V. Prentice Hall (2002) ISBN 0-201-44494-7

11. Kumar, S., Kumar, P.R.: Queueing Network Models in the Design and Analysis of Semiconductor Wafer Fabs. IEEE Transactions on Robotics and Automation 17(5), 548-561 (2001)

12. Jackson, J.R.: Networks of waiting lines. Operations Research 5(4) (1957) 
13. Baskett, F., Chandy, K.M., Muntz, R.R., Palacios, F.G.: Open, Closed, and Mixed Networks of Queues with Different Classes of Customers. Journal of the Association for Computing Machinery 22(2), 248-260 (1975)

14. Wolff, R.W.: Stochastic Modeling and the Theory of Queues, 1st edn., pp. 321-322. Prentice Hall (1989)

15. Kuehn, P.J.: Approximate Analysis of General Queueing Networks by Decomposition. IEEE Transactions on Communications Com. 27(1) (1979)

16. Hu, S.-C., Chuah, Y.K.: Power consumption of semiconductor fabs in Taiwan. Energy 28(8), 895-907 (2003)

17. Whitt, W.: The Queueing Network Analyzer. The Bell System Technical Journal 62(9) (1983)

18. Daley, D.J.: Queueing Output Processes. Adv. Appl. Prob. 8, 295-415 (1976) 